\title{
Surfactant-free polymeric nanoparticles for biomedical applications
}

\author{
Crucho, C.I.C.* and Barros, M.T.* \\ * REQUIMTE/CQFB, Departamento de Química, Faculdade de Ciências e Tecnologia, Universidade Nova \\ de Lisboa, 2829-516 Caparica, Portugal \\ Email: cic17468@ campus.fct.unl.pt
}

Nature continues to be the ultimate in nanotechnology, where polymeric nanoarchitectures play a central role in biological systems. Inspired by the way nature forms functional supramolecular assemblies, polymeric nanoparticles (PNPs) have emerged as promising nanomaterials for drug delivery due to their ability to overcome multiple biological barriers and tailorability. In the present day scenario, nanomedicine embraces many opportunities arising from chemical functionalization. Thus, stability, drug release and targeting can be tailored by surface modification, improving the pharmacological profiles of therapeutic molecules [1].

In this work new amphiphilic polymers were designed and synthesized by chemical modification of poly(ethylene glycol) (PEG) conjugated with sucrose and cholic acid (abbreviated as Suc-PEG-Chol). In this system, cholic acid will act as a drug incorporation site and sucrose as targeting moiety. Due to its hydrophilic nature PEG provides protection from blood proteins adsorption and reduces the uptake by the mononuclear phagocyte system. Three series of polymers with different PEG chain lengths were synthesized using methods based upon our experience in carbohydrate chemistry $[2,3]$. Investigations using pyrene as a fluorescence probe showed that the Suc-PEG-Chol conjugate may self-assemble in water to form core-shell structure nanoparticles. The critical association concentration (CAC) value was found to be in the range of $0.06-0.13 \mathrm{~g} / \mathrm{L}$ and was dependent on the PEG block length.

Owing to their amphiphilic characteristics in aqueous solution, PNPs of Suc-PEG-Cholic conjugates were prepared by nanoprecipitation method without any surfactants. The physicochemical characteristics of the obtained colloidal suspension of PNPs were determined by dynamic light scattering (DLS). The mean particle size diameters was $117 \mathrm{~nm}$ for Suc-PEG ${ }_{2000}-$ Chol conjugate and 96 and $111 \mathrm{~nm}$ for the PEG $_{4000}$ and $\mathrm{PEG}_{6000}$ analogs respectively, which is suitable for drug delivery systems. [4] All the obtained PNPs presented a narrow particle size distribution (PDI < 0.3), negative surface charge and no size dependence on the polymer concentration, forming stable nanoparticle suspensions.

From the atomic force microscopy (AFM) observations, PNPs exhibited a spherical shape with a relatively smooth surface. However, the average diameters determined by AFM were higher than those measured by DLS. The lyophilized PNPs also exhibit the same behavior. From these results we noticed some influence from the water evaporation entailed in the preparation of the AFM sample on the size of the PNPs. [5] Scanning electron microscopy (SEM) was also used to study the surface morphology of the PNPs with higher resolution. The lyophilized Suc- $\mathrm{PEG}_{4000}$-cholic PNPs size found from the SEM images tallies with that detected by DLS previous to lyophilization which indicates that no aggregation occurred during the lyophilization process even without any cryoprotectant.

Although further investigation on the in vitro and in vivo effect of Suc-PEG-Cholic PNPs is required, our results suggest that these PEGylated nanoparticles formulated with cholic acid and sucrose as biocompatible building blocks can be considered a potential candidate for biomedical applications.

This work has been supported by Fundação para a Ciência e a Tecnologia (FCT) through grant no. PEstC/EQB/LA0006/2013. Carina I. C. Crucho thanks to FCT for a PhD grant (SFRH/ BD/71648/ 2010) and to Prof. Dr. Jorge Caldeira for his valuable scientific support in the introduction and use of AFM. The authors thank Eng. Isabel Nogueira (MicroLab, IST) for the SEM images. 
[1] Duncan R., Current Opinion in Biotechnology, 22, 492-501, 2011.

[2] Barros M.T. et al., Journal of Organic Chemistry, 69, 7772-7775, 2004.

[3] Crucho C.I.C. et al., Molecules, 13, 762-770, 2008.

[4] Iversen T.-G. et al., Nano Today, 6, 176-185, 2011.

[5] Rao A. et al, Journal of Physics: Conference Series., 61, 971-976, 2007.

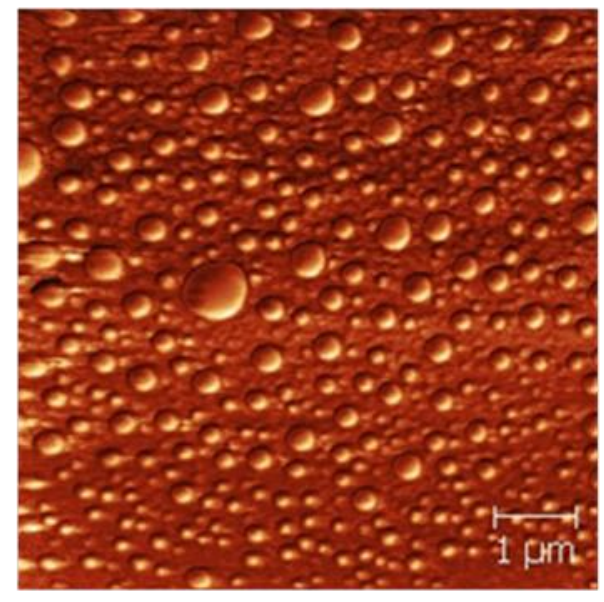

Figure 1 - AFM phase image of lyophilized Suc- $\mathrm{PEG}_{2000}$-Chol PNPs $(0.1 \mathrm{mg} / \mathrm{mL})$.

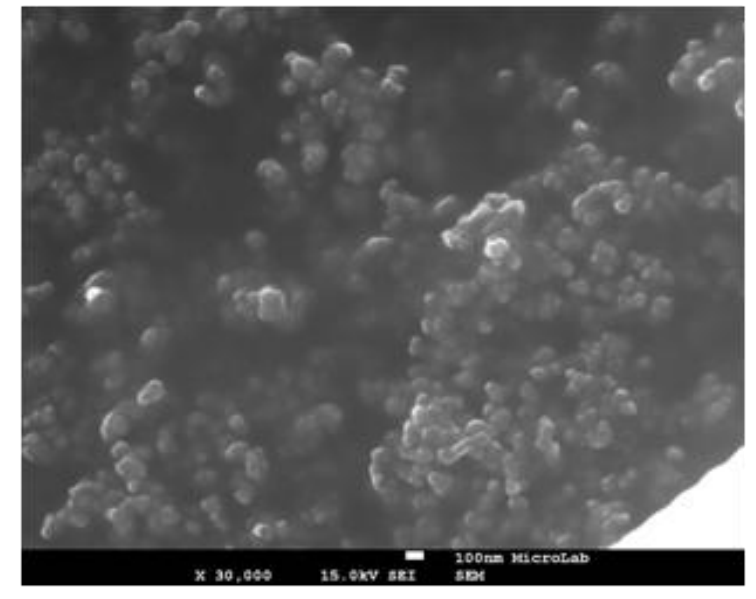

Figure 2 - SEM image of Suc-PEG $4000-$ Chol nanoparticles. 